\title{
The assessment of cardiovascular reflex activity: standardization is needed
}

\author{
W. Wieling and J. J.van Lieshout \\ Department of Medicine, Academic Medical Centre, Amsterdam, The Netherlands
}

Perturbing the circulatory system and watching its subsequent recovery is the basis of testing cardiovascular reflex control. The intriguing process of regaining balance by alteration of para- and orthosympathetic activity provides useful information about integrity and effectiveness of short-term autonomic circulatory control. Both the autonomic nervous system and circulatory haemodynamics are involved; impaired cardiovascular reflex activity can, therefore, not simply be equated to a lesion in autonomic nerves.

Cardiovascular reflex activity can be assessed by a large number of tests. In 1982 Ewing and coworkers introduced a test battery for studying autonomic damage in diabetic patients consisting of five tests, i.e. the heart rate responses induced by forced breathing, the Valsalva manoeuvre and standing up and the changes in blood pressure induced by standing and handgrip [1]. In this issue of Diabetologia Ryder and Hardisty propose a modification of the Ewing test battery based upon a study performed by O'Brien and colleagues [2]. The proposed test battery is claimed to be simpler and quicker. Ryder and Hardisty's argument that testing can be simplified by performing only one Valsalva manoeuvre instead of three is supported by a recent study from Finland [3]. The use of one deep breath instead of six to test cardiac vagal control is, however, not supported by that investigation; it recommends conduction of the forced breathing test by performing three deep breaths [3]. This issue needs further study. The test battery proposed by Ryder and Hardisty is not a particularly quick test; 10 to $20 \mathrm{~min}$ of supine rest are required prior to the standing up test [4]. The strongest argument in favour of the use of O'Brien's data in studies of autonomic neuropathy is that the age related normal ranges for cardiac vagal control are based on a large number (310) of normal subjects over a wide age range (18-85 years). Ewing uses only one normal range regardless of age. The use of a single limit for all ages will result in errors of diagnosis $[5,6]$. Furthermore, we agree with Ryder and Hardisty that the initial heart rate response upon standing should be quantified by the HRmax/HRmin ratio and not by the $30 / 15$ ratio as used in the Ewing test battery $[5,6]$.

O'Brien and colleagues deserve credit for their work. Nevertheless, we have published several comments [4]. Slightly lower limits were obtained compared to previous studies. This is attributed by O'Brien et al. to the larger number and more uniform age distribution of the subjects in their study [2]. Differences in selection criteria and test conditions are, in our view, a more likely explanation [4]. In O'Brien's studies no restrictions were imposed on the consumption of coffee or cigarette smoking. Subjects were tested between 09.00 hours and 17.00 hours but the relationship to with meals is not reported. No data are given on room temperature, nor on baseline heart rate or blood pressure [2]. It is well documented that these factors have considerable influence on tests that are aimed at assessment of autonomic circulatory control. Furthermore, O'Brien's data almost exclusively deal with heart rate variability, whereas blood pressure control is assessed only by the change in systolic blood pressure upon standing. Yet, it is not a fall in systolic, but in diastolic pressure upon standing that is typical of autonomic failure [5]. In tests for autonomic neuropathy it is mandatory to take age into account [6]. Two other factors have been shown to have a comparable influence on the cardiovascular reflexes involved, i.e. length of the period of supine rest prior to the standing up test and body position during the Valsalva manoeuvre (Fig. 1) [7]. We recommend performing the standing up test after 5-10 min supine rest, a very short ( $1 \mathrm{~min}$ ) resting period as used in the Ewing test battery will yield relatively small circulatory responses, the very long resting period used by O'Brien is unnecessary. We prefer, like Ewing, to have the Valsalva manoeuvre performed in the sitting position, because the circulatory effects are larger than in the supine position that O'Brien uses. Finally, we advise conducting the forced breathing test, like O'Brien but unlike Ewing, in the supine position, since vagal effects are then most pronounced. 

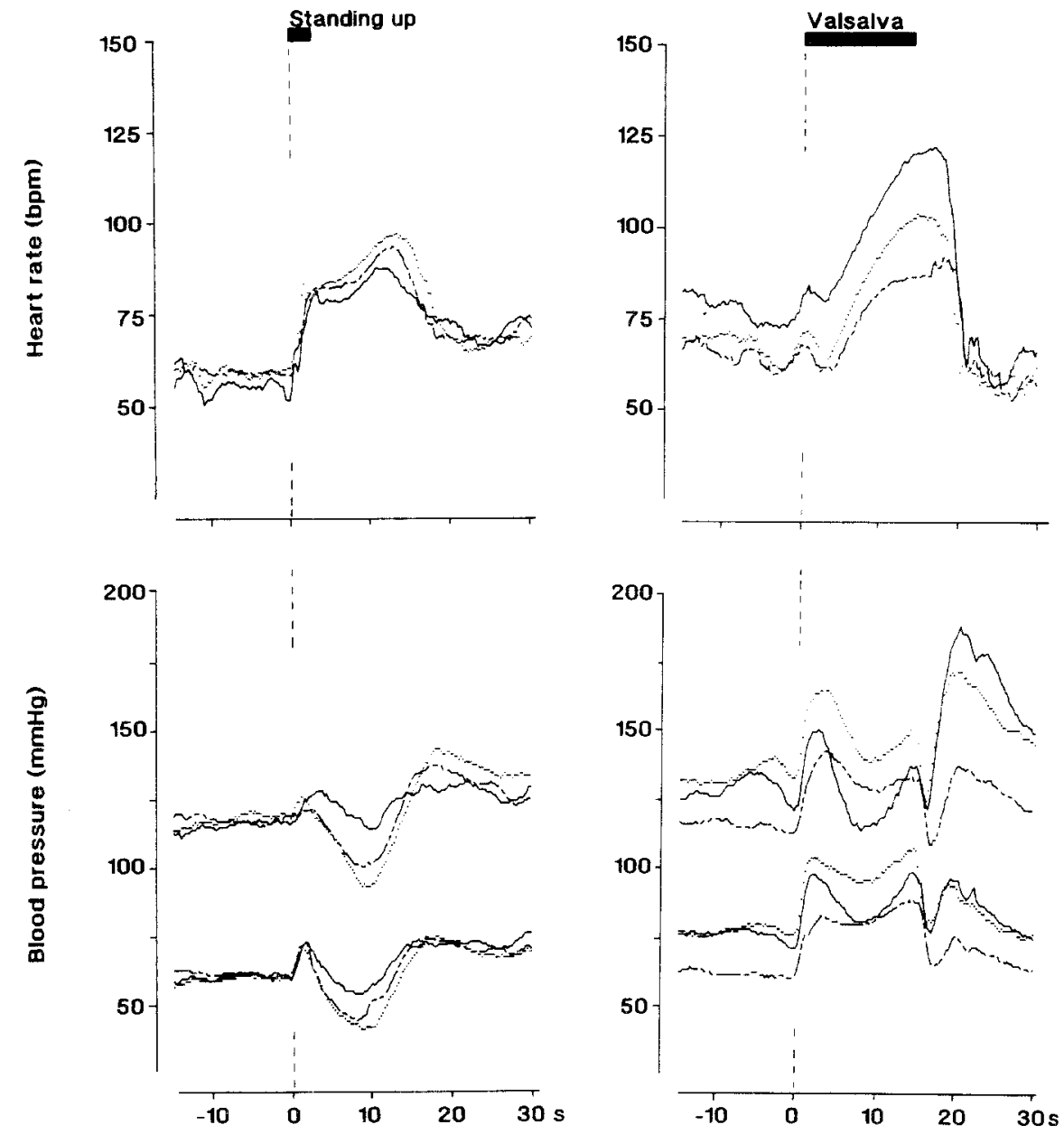

Fig. 1. Left panel. Influence of period of preceding rest on the blood pressure and heart rate responses to standing.

$-1 \mathrm{~min}$ of preceding rest;

$\ldots \ldots-\ldots$ min of preceding rest:

$\ldots \ldots 20$ min of preceding rest. Bar marked "standing up" indicates the duration of the change of posture. Right panel. Influence of posture on the blood pressure and heart rate responses to the Valsalva manoeuvre. _ _ - - - after 5 min supine; ..... . after 5 min sitting; —_ after 20 min standing. Bar marked "Valsalva" indicates the duration of the straining period. From Ten Harkel et al. [7] with permission from the American Physiological Society
Ryder and Hardisty are critical about test procedures, but leave their readers to judge the utility of the battery itself and the indications for its application. Measurement of cardiovascular reflex activity is useful in evaluation of patients suspected as suffering from autonomic neuropathy and in specific studies on autonomic neuropathy, but there is no indication for routine testing in clinical practice. We agree that there is a real need for standardization of tests. The suggestions by Ryder and Hardisty are an important step forward towards that goal, but are not sufficiently conclusive and simple enough to merit general endorsement.

\section{References}

1. Ewing DJ, Clarke BF (1982) Diagnosis and management of diabetic neuropathy. Br Med J 285: 916-918

2. O'Brien IAD, O'Hare P, Corrall RJM (1982) Heart rate variability in healthy subjects: effects of age and the derivation of normal ranges for test of autonomic function. Br Heart J 55:348-354
3. Mustonen J, Länsimies E, Uusitupa, Talwar S, Hyödynmaa S, Kärkkäinen A (1989) Testing of cardiovascular regulation-methodological considerations. Clin Physiol 9:249-257

4. Wieling W (1987) Heart rate variability in healthy subjects: effect of age and derivation of normal ranges for tests of autonomic function. Br Heart J 57: 109-110

5. Wieling W (1988) Standing, orthostatic stress and autonomic function. In: Bannister R (ed) Autonomic failure. A textbook of clinical disorders of the autonomic nervous system. Oxford University Press, Oxford, pp 308-320

6. Wieling W, Van Brederode JFM, De Rijk LG, Borst C, Dunning AJ (1982) Reflex control of heart rate in normal subjects in relation to age: a data base for cardiac vagal neuropathy. Diabetologia 22: 163-166

7. Ten Harkel ADJ, Van Lieshout JJ, Van Lieshout EJ, Wieling W (1990) The assessment of cardiovascular reflexes; influence of posture and period of preceding rest. J Appl Physiol 68: 147-153

Dr. W. Wieling

Department of Medicine F4

Academic Medical Centre

Meibergdreef 9

NL-1105 AZ Amsterdam

The Netherlands 